\title{
Surface functionalization of the honeycomb structure of zinc antimonide (ZnSb) monolayer: A first-Principles study
}

\author{
A. Bafekry ${ }^{\mathrm{a}, \mathrm{b}}$, M. Shahrokhi ${ }^{\mathrm{c}}, \mathrm{M}$. Yagmurcukardes ${ }^{\mathrm{d}}$, D. Gogova ${ }^{*}, \mathrm{e}, \mathrm{M}$. Ghergherehchi $^{\mathrm{f}}$, \\ B. Akgenc ${ }^{g}$, S.A.H. Feghhi ${ }^{\text {b }}$ \\ ${ }^{a}$ Department of Physics, University of Antwerp, Groenenborgerlaan 171, B-2020 Antwerp, Belgium \\ ${ }^{\mathrm{b}}$ Department of Radiation Application, Shahid Beheshti University, G. C., Tehran, Iran \\ ${ }^{\mathrm{c}}$ Department of Physics, Faculty of Science, University of Kurdistan, 66177-15175 Sanandaj, Iran \\ d Department of Photonics, Izmir Institute of Technology, 35430 Izmir, Turkey \\ e Department of Physics, University of Oslo, P.O. Box 1048, Blindern, Oslo, Norway \\ ${ }^{\mathrm{f}}$ College of Electronic and Electrical Engineering, Sungkyun Kwan University, Suwon, Korea \\ ${ }^{g}$ Department of Physics, Kirklareli University, Kirklareli, 39100, Turkey
}

\section{A R T I C L E I N F O}

\section{Keywords:}

Zinc antimonide (znsb)

2D Materials

Electro-optic properties

Functionalization

First-Principles calculations

\begin{abstract}
A B S T R A T
Structural, electronic, optic and vibrational properties of Zinc antimonide (ZnSb) monolayers and their functionalized (semi-fluorinated and fully chlorinated) structures are investigated by means of the first-principles calculations. The phonon dispersion curves reveal the presence of imaginary frequencies and thus confirm the dynamical instability of $\mathrm{ZnSb}$ monolayer. The calculated electronic band structure corroborates the metallic character with fully-relativistic calculations. Moreover, we analyze the surface functionalization effect on the structural, vibrational, and electronic properties of the pristine ZnSb monolayer. The semi-fluorinated and fullychlorinated ZnSb monolayers are shown to be dynamically stable in contrast to the ZnSb monolayer. At the same time, semi-fluorination and fully-chlorination of ZnSb monolayer could effectively modulate the metallic electronic properties of pristine $\mathrm{ZnSb}$. In addition, a magnetic metal to a nonmagnetic semiconductor transition with a band gap of $1 \mathrm{eV}$ is achieved via fluorination, whereas a transition to a semiconducting state with $1.4 \mathrm{eV}$ band gap is found via chlorination of the $\mathrm{ZnSb}$ monolayer. According to the optical properties analysis, the first absorption peaks of the fluorinated- and chlorinated-ZnSb monolayers along the in-plane polarization are placed in the infrared range of spectrum, while they are in the middle ultraviolet for the out-of-plane polarization. Interestingly, the optically anisotropic behavior of these novel monolayers along the in-plane polarizations is highly desirable for design of polarization-sensitive photodetectors. The results of the calculations clearly proved that the tunable electronic properties of the $\mathrm{ZnSb}$ monolayer can be realized by chemical functionalization for application in the next generation nanoelectronic devices.
\end{abstract}

\section{Introduction}

The rise of two-dimensional (2D) ultra-thin materials has started by the successful synthesis of graphene[1]. Following graphene, many different 2D materials (2DMs) have been demonstrated and added to the ultra-thin materials family [2-23] exhibiting unique electronic, optical, and mechanical properties. Recently, the effort on the synthesis of 2D structures of non-layered bulk materials has increased exponentially. With this regard, the 2D forms of $\mathrm{Cr}_{2} \mathrm{~S}_{3}$ [24], hematite[25,26], gallenene [27], and very recently $\mathrm{ZnSb}[28]$ have been demonstrated using different experimental techniques. Among the non-layered bulk materials, ZnSb has been extensively studied due to it promising thermoelectric properties [29-34]. Zn-Sb exhibits two stable phases, namely $\mathrm{ZnSb}$ and $\beta-\mathrm{Zn}_{4} \mathrm{Sb}_{3}$ at room temperature $[35,36]$. The $\beta-\mathrm{Zn}_{4} \mathrm{Sb}_{3}$ phase has been reported to possess a low thermal conductivity due to disordered $\mathrm{Zn}$ atoms in its crystalline structure[37-39]. In a recent experiment, a 2D form of the $\mathrm{ZnSb}$ phase has been demonstrated as a new member of 2D family [28]. Synthesis of $\mathrm{ZnSb}$ ultra-thin layers has been been realized by a change of the hybridization from $s p^{3}$ to $s p^{2}$.

The confinement of electrons in one-dimension makes the 2DMs suitable candidates for surface functionalization which can significantly modify tune their electronic and optical properties. The theoretical

\footnotetext{
* Corresponding author.

E-mail addresses: bafekry.asad@gmail.com (A. Bafekry), mitragh@skku.edu (M. Ghergherehchi).
} 
predictions and experimental observations revealed that electronic properties of graphene can be tuned via either one or two types of surface modifications [40-43]. In addition, fluorination has been shown to be also an efficient way for tuning the structural, magnetic, electronic, and mechanical properties of 2DMs [44-50]. In addition to free-standing stable 2DMs, the 2D forms of non-layered bulk materials offer a rich variety of advantages for their surface functionalization due to the unsaturated surface orbitals. It was revealed theoretically that the surface functionalization may lead to dynamical stability of the 2D layer, synthesized from a non-layered bulk material, as a free-standing crystal [28].

Surface adsorption on 2DMs using different types of adatoms such as $\mathrm{H}, \mathrm{F}, \mathrm{Cl}$, or Li have been experimentally demonstrated. Amongst all the atoms, the $\mathrm{F}$ is one of the most important adatoms because its incorporation into the structure gives rise to significant structural, magnetic, electronic, and mechanical changes[51-62] as a result of its high electronegativity. In the first experimental realization of fluorinated graphene, it was investigated that constructed fluorographene is an insulator at room temperature and it keeps its stability till $400^{\circ} \mathrm{C}$ [57]. In addition to the $\mathrm{F}$ adsorption, manipulation of 2DMs by employing $\mathrm{Cl}$ atoms were also widely studied. For instance, chlorination of graphene surfaces with different compositions and configurations was investigated theoretically and it was figured out that the stable structures of chlorinated graphene were significantly determined by the coverage amount $[63,64]$. Apart from the theoretical predictions, chlorination scenarios were also demonstrated experimentally and several studies reported different amounts of chlorination in graphene, such as $2 \%$ [65], $8 \%[66]$, and $25 \%$ [67]. Apparently, the present experimental techniques allow researchers to controllably functionalize the surfaces of 2DMs in order to tune their properties.

Motivated by the recent progress in non-layered 2DMs, their stability engineering and experimental possibilities of surface functionalization, we explore the effect of one and two surface functionalization of a single-layer $\mathrm{ZnSb}$ via fluorination and chlorination, respectively. Our results reveal that dynamically unstable free-standing $\mathrm{ZnSb}$ sheet can be stabilized via either one-surface fluorination or by two-surface chlorination. In addition to the enhanced dynamical stability, we have discovered that the metallic ZnSb single-layer turns into a semiconductor in both cases of surface functionalization.

\section{Computational methodology}

The structural optimization and electronic properties calculations were performed by the plane-wave basis projector augmented wave (PAW) method in the framework of density-functional theory (DFT). The generalized gradient approximation (GGA) with the Perdew-BurkeErnzerhof(PBE) $[68,69]$ functional was used for the exchange and correlation contributions as implemented in the Vienna ab-initio Simulation Package (VASP)[70,71]. Moreover, a spin-orbit-coupling (SOC) effect was included on the top of the GGA and Heyd-Scuseria-Ernzerhof (HSE06)[72] screened-nonlocal-exchange functional of the generalized Kohn-Sham scheme to get more accurate band gap calculations. To describe the long-range van der Waals (vdW) interactions, the vdW correction DFT-D2 proposed by Grimme was utilized[73]. The Bader technique is used for analysis of the charge transfers in the structures [74]. The kinetic energy cut-off for plane-wave expansion was set to 500 $\mathrm{eV}$ and the energy was minimized until its variation in the following steps became $10^{-8} \mathrm{eV}$. To get the optimized structures, the total Hellmann-Feynman forces were reduced to $10^{-7} \mathrm{eV} / \AA . \AA .21 \times 21 \times 1 \Gamma$ centered $k$-point sampling was used or the primitive unit cells by using the Monkhorst-Pack [75]. The vibrational properties were obtained from the small displacement method as implemented in the PHON code [76]. Ab initio molecular dynamics (AIMD) simulations were also carried out to examine the thermal stability of functionalized $\mathrm{ZnSb}$ single-layers by using $4 \times 4 \times 1$ super cells at room temperature $(300 \mathrm{~K})$

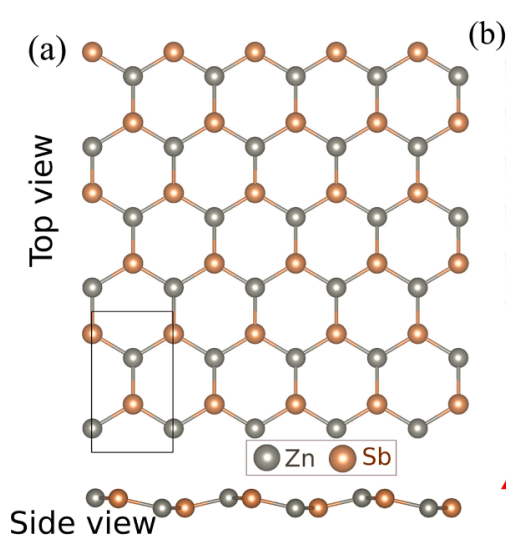

(b)
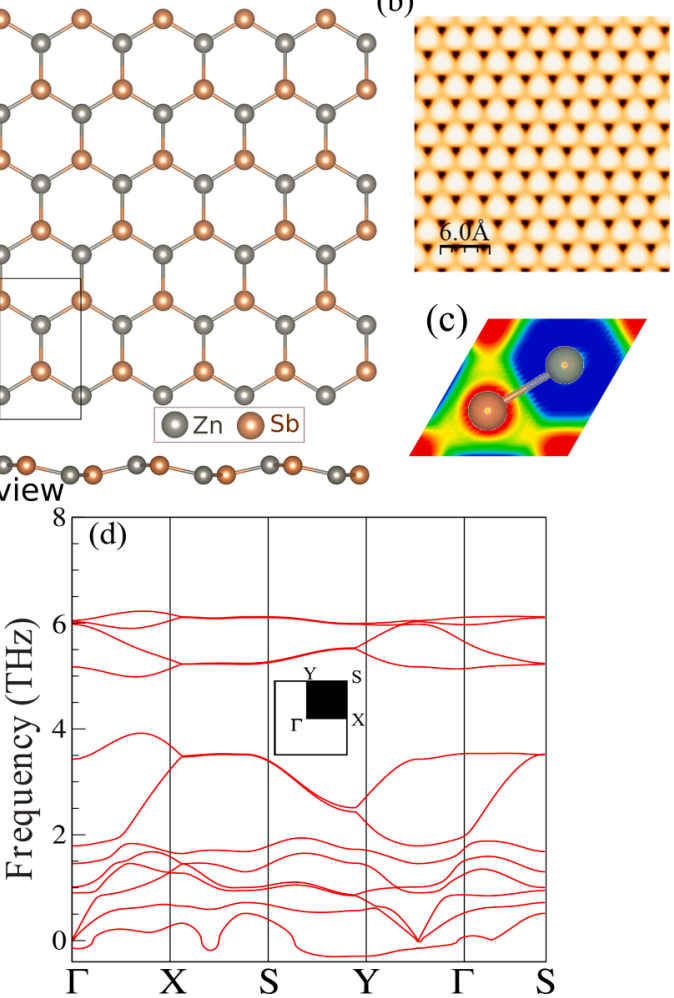

Fig. 1. (a) Atomic structure of $\mathrm{ZnSb}$ monolayer. With the gray (orange) representing $\mathrm{Zn}(\mathrm{Sb})$ atoms. The primitive unit cell by a dark rectangular. (b) Simulated STM image. (c) Top view of the electron localization function (ELF) isosurface from 0.0 (blue) to 1.0 (red) and (d) Phonon band structure for $\mathrm{ZnSb}$ monolayer. (For interpretation of the references to colour in this figure legend, the reader is referred to the web version of this article.)

with total simulation time of 6 ps with 2 fs time steps. The optical calculations were performed in the random phase approximation (RPA) [77] method constructed over the screened hybrid HSE06 functional using the VASP code.

\section{Pristine monolayer znsb}

The slightly buckled honeycomb atomic structure of $\mathrm{ZnSb}$ monolayer which has a tetragonal primitive unit cell, formed by two $\mathrm{Zn}$ and two $\mathrm{Sb}$ atoms, the $\mathbf{a}$ and $\mathbf{b}$ are equal vectors that make a $90^{\circ}$ angle, exhibits $P_{3 m 1}$ space group, as shown in Fig. 1(a). The optimized structures are obtained in such a away that all the atomic positions and the lattice vectors are fully relaxed. The optimized lattice constants are equal to 4.49 and $7.48 \AA$, respectively, while the bond lengths are determined to be $2.60 \AA$. A simulated STM image of the ZnSb monolayer is shown in Fig. 1(b). The atomistic structure is easily recognised from the predicted STM image, where the $\mathrm{Sb}$ atoms are brighter than the $\mathrm{Zn}$ atoms. The electron localization function is illustrated in Fig. 1(c). The difference charge density $(\Delta \rho)$ is calculated using the following equation

$\Delta \rho=\rho_{Z n S b}-\rho_{Z n}-\rho_{S b}$

where $\rho_{Z n S b}, \rho_{Z n}$ and $\rho_{S b}$ represents the charge densities of the $\mathrm{ZnSb}$ monolayer and isolated atoms, respectively. The result of difference charge density calculations gives the positively charged $\mathrm{Zn}$ atoms are surrounded by negatively charged $\mathrm{Sb}$ atoms. Based on the charge transfer analysis, we found out that the $\mathrm{Sb}$ atoms gain as $0.16 e$ from the adjacent $\mathrm{Zn}$ atoms. According to the Pauling electronegativity scale, $\mathrm{Sb}$ (2.18) atom has a larger electronegativity than Zn (1.65) and it results in large difference of electron density. The cohesive energy per atom that is quantifying the stability of materials is calculated using the following 
Table 1

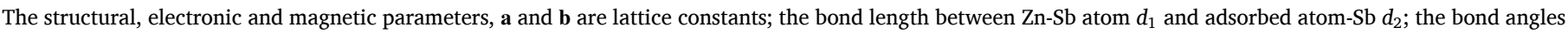

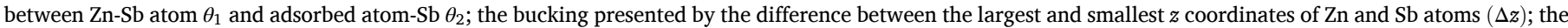

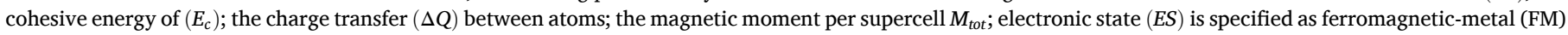
and semiconductor (SC). The band gap $\left(E_{\text {gap }}\right)$ within GGA+SOC and HSE06 are placed outside and inside the closing parenthesis.

\begin{tabular}{|c|c|c|c|c|c|c|c|c|c|c|c|c|}
\hline & $\begin{array}{l}a \\
(\AA)\end{array}$ & $\begin{array}{l}b \\
(\AA)\end{array}$ & $\begin{array}{l}d_{1} \\
(\AA)\end{array}$ & $\begin{array}{l}d_{2} \\
(\AA)\end{array}$ & $\begin{array}{l}\Delta z \\
(\AA)\end{array}$ & $\begin{array}{l}\theta_{1} \\
\left(^{\circ}\right)\end{array}$ & $\begin{array}{l}\theta_{2} \\
\left(^{\circ}\right)\end{array}$ & $\begin{array}{l}E_{c} \\
\text { (eV/atom) }\end{array}$ & $\begin{array}{l}\Delta Q \\
(e)\end{array}$ & $\begin{array}{l}M_{t o t} \\
\left(\mu_{B}\right)\end{array}$ & $E S$ & $\begin{array}{l}E_{\text {gap }} \\
(\mathrm{eV})\end{array}$ \\
\hline $\mathrm{ZnSb}$ & 4.49 & 7.48 & 2.60 & - & 0.59 & 120 & - & 1.41 & 0.16 & 1.3 & M & - \\
\hline Fluorinated-ZnSb & 4.66 & 7.78 & 2.69 & 2.03 & 0.53 & 117 & 95 & 2.41 & 0.36 & 0 & SC & $0.06(1.0)$ \\
\hline Chlorinated-ZnSb & 4.63 & 7.76 & 2.64 & 2.54 & 0.89 & 117 & 65 & 2.02 & 0.50 & 0 & SC & $0.50(1.4)$ \\
\hline
\end{tabular}

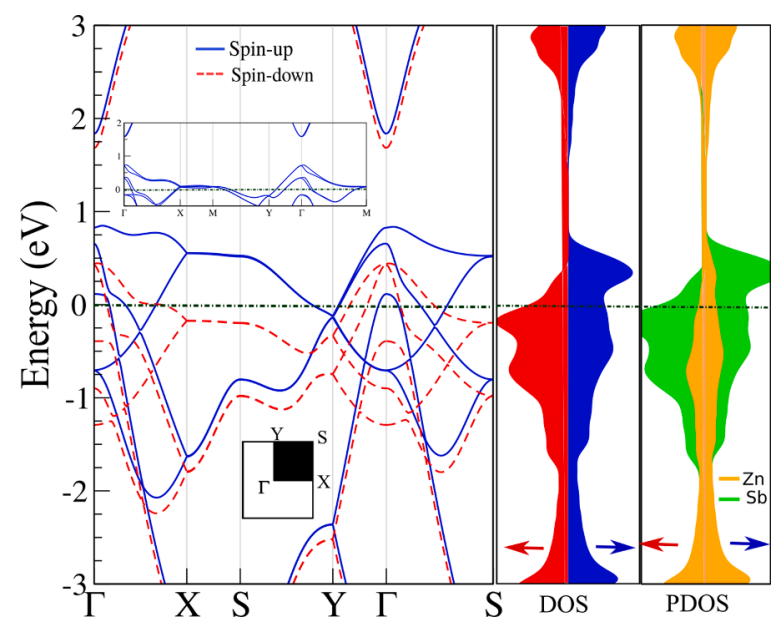

Fig. 2. (a) Electronic band structure, DOS and PDOS of $\mathrm{ZnSb}$ monolayer without corresponding SOC. Band structure with SOC is shown in the inset. Fermi level is set to zero.

equation:

$E_{c o h}=\left[\left(n_{Z n} E_{Z n}+n_{S b} E_{S b}\right)-E_{Z n S b}\right] /\left(n_{Z n}+n_{S b}\right)$,

where $E_{Z n}$ and $E_{S b}$ represent the energies of isolated single $\mathrm{Zn}$ and $\mathrm{Sb}$ atoms; $E_{Z n S b}$ represents the total energy of the ZnSb monolayer. In addition, $\mathrm{n}_{Z n}$ and $\mathrm{n}_{S b}$ stand for the number of $\mathrm{Zn}$ and $\mathrm{Sb}$ atoms in the primitive unitcell, respectively. The cohesive energy of the $\mathrm{ZnSb}$ monolayer is found to be $1.41 \mathrm{eV} /$ atom. The dynamical stability is examined by calculating its phonon band dispersions and is presented in Fig. 1(d). The phonon branches are almost free from any imaginary frequencies, except the out-of-plane acoustic phonon branch which has completely negative frequencies through the whole BZ indicating the a dynamical instability of the structure. Our results confirm that the pristine $\mathrm{ZnSb}$ does not show dynamical stability. The existence of negative frequencies for the out-of-plane acoustical phonon branch reveals that the unoccupied orbitals of the individual atoms need to be saturated in order to possess the stability. The results of the electronic structure calculations, i.e., the density of states (DOS) and partial DOS (PDOS) of the $\mathrm{ZnSb}$ monolayer are presented in Fig. 2. The band structure with SOC [78] is depicted in the inset. Our computational results show that $\mathrm{ZnSb}$ is a ferromagnetic-metal material with valence bands crossing the Fermi level and it has $1.3 \mu_{B}$ magnetic moment in the ground state. Note, that the metallic characteristic is preserved when the SOC is included. From the DOS and PDOS as shown in Fig. 2, it is clearly seen that the bands around the Fermi level are composed of Sb- $p_{z}$ and Zn- $d_{x z, y z}$ orbitals.

\section{Functionalization of a znsb monolayer}

Adsorption is one of the efficient ways to tune the structural, electronic, and magnetic properties of 2DMs. Halide atoms such as $\mathrm{F}$ and $\mathrm{Cl}$
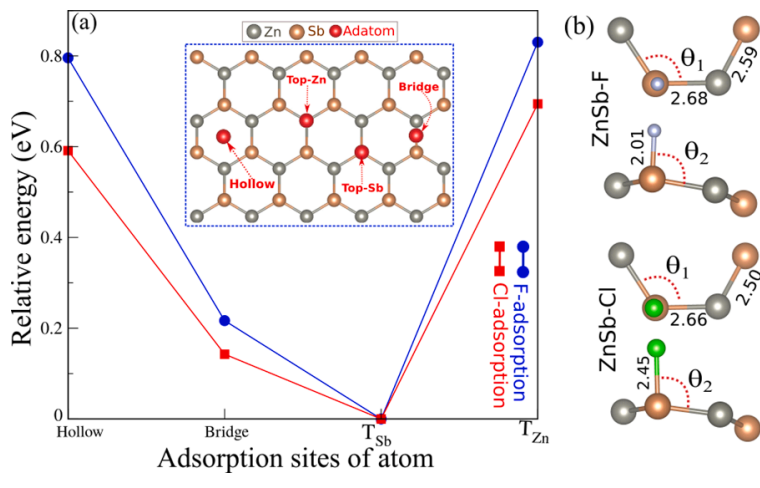

Fig. 3. (a) Relative total energies of four possible adsorption sites in $\mathrm{ZnSb}$ monolayer. The inset shows top views of four possible adsorption sites. (b) optimized structures with corresponding structural parameter including bond length of $\mathrm{ZnSb}$ with adsorption of $\mathrm{F}$ and $\mathrm{Cl}$ atoms.

are potential candidates for such functionalizations. Up to now, fluorinated-graphene (F adsorption on graphene) was synthesized and its numerous physical properties were predicted also theoretically. The ground state structures are obtained by placing a single $\mathrm{F} / \mathrm{Cl}$ atom on

(a)
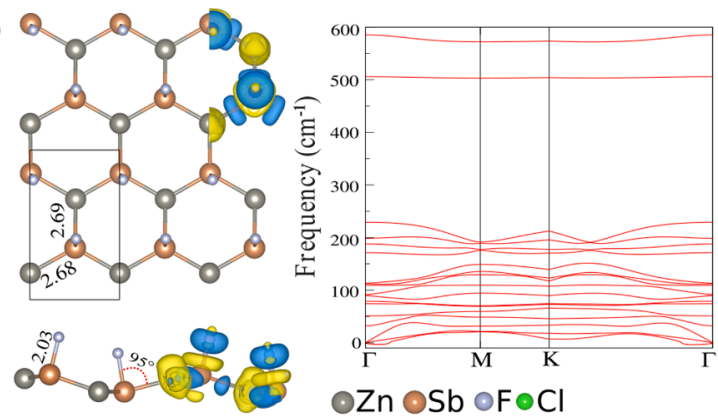

(b)
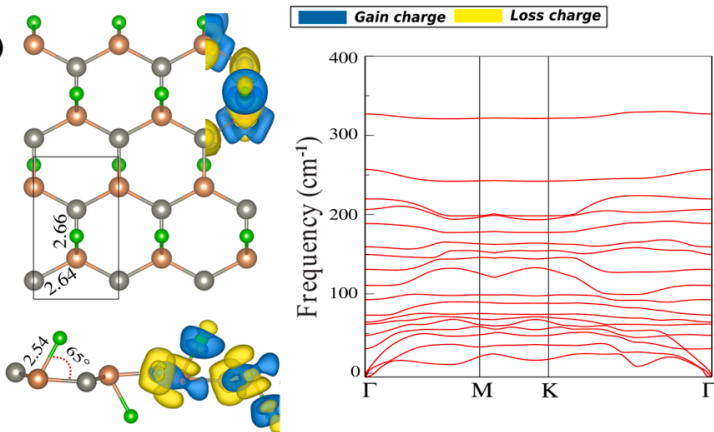

Fig. 4. Atomic structures with corresponding structural parameters (left) and phonon dispersion (right) of (a) fluorinated-ZnSb and (b) chlorinated-ZnSb with difference charge density isosurfaces. Charge accumulation and depletion regions are represented by blue and yellow colors, respectively. (For interpretation of the references to colour in this figure legend, the reader is referred to the web version of this article.) 
four preferable for adsorption sites as shown in the inset of Fig. 3. Different adsorption sites of $\mathrm{F}$ and $\mathrm{Cl}$ on $\mathrm{ZnSb}$ given with respect to the position; (a) hollow (above the center of a hexagon with six $\mathrm{Zn}$ and $\mathrm{Sb}$ atoms $\left(H_{Z n S b}\right)$ ), (b) bridge (above the middle of a Zn-Sb bond $\left(B_{Z n S b}\right)$ ), (c) top-Zn (top site above a $\mathrm{Zn}$ atom $\left(T_{Z n}\right)$ ), (e) top-Sb (the top site above a $\mathrm{Sb}$ atom $\left(T_{S b}\right)$ ). The most feasible site is selected from four possible adsorption sites according to the relative total energies depicted in Fig. 3 (a). The inset shows top views of the four possible adsorption sites. We have determined the most stable adsorption site for $\mathrm{F}$ and $\mathrm{Cl}$ atoms is the $\left(T_{S b}\right)$ site. The optimized structures and the corresponding structural parameters of monolayers with adsorbed $\mathrm{F}$ and $\mathrm{Cl}$ are presented in Fig. 3 (b). As the single $\mathrm{F}$ and $\mathrm{Cl}$ atoms are adsorbed on the surface through the $\mathrm{Sb}$ atom, there occurs a small distortion in the lattice along the bond direction. The bond lengths for $\mathrm{F}-\mathrm{Sb}$ and $\mathrm{Cl}-\mathrm{Sb}$ interactions are calculated to be 2.01 and $2.45 \AA$, respectively. As compared to their adsorption energies on the hollow, bridge, and top of the $\mathrm{Zn}$ atom, the top of the $\mathrm{Sb}$ atom is significantly favourable site for the adsorption of $\mathrm{F}$ and $\mathrm{Cl}$ atoms.

$E_{\text {adsorp }}=\left(E_{\text {supercell }}+E_{\text {adatom }}\right)-E_{\text {adsorbed }}$

where $\mathrm{E}_{\text {supercell }}$ and $\mathrm{E}_{\text {adsorbed }}$ stand for the total energies of bare $\mathrm{ZnSb}$ supercell and the single-atom adsorbed monolayers while $\mathrm{E}_{\text {adatom }}$ represents the total energy of an isolated adatom ( $\mathrm{F}$ or $\mathrm{Cl}$ ). Once the favorable adsorption sites of single $\mathrm{F}$ and $\mathrm{Cl}$ atoms are examined, the possible full-functionalization of $\mathrm{ZnSb}$ is considered in two different configurations. In the first scenario, all Sb atoms are saturated by either $\mathrm{F}$ or $\mathrm{Cl}$ atoms from one surface. The atomic structures with corresponding structural parameter including bond length and bond angle are shown in Figs. 4(a,b). The difference charge density is given in the same panel in Fig. 4. The structural parameters such as lattice constant, the bond lengths between atoms are summarized in Table I. Notice that, the lattice constants and the bond lengths between Sb-Zn change via the functionalization of the $\mathrm{ZnSb}$ monolayer. In fluorinated-ZnSb and chlorinated-ZnSb an a increase of the lattice constant a appears from $4.49 \AA$ in pristine $\mathrm{ZnSb}$, to 4.66 and $4.63 \AA$, respectively. The increase of the lattice constant is due to the increase in bond lengths, which is changed from 2.60 to $2.69 \AA$ (fluorinated) and to $2.64 \AA$ (chlorinated). In addition, the Sb-F and Sb-Cl bond lengths adsorption of fluorinated$\mathrm{ZnSb}$ and chlorinated-ZnSb are calculated as 2.03 and $2.54 \AA$, respectively. From the difference charge density it is seen that because of the strong bonding between $\mathrm{Sb}$ and $\mathrm{Cl}$ atoms, a charge accumulation occurs in the region between the $\mathrm{Cl}$ atom and the neighboring $\mathrm{Sb}$ atoms. Based on the Bader charge analysis, in fluorinated- $\mathrm{ZnSb}$ each $\mathrm{F}$ atom gains about $0.36 e$, while in chlorinated- $\mathrm{ZnSb}$, each $\mathrm{Cl}$ atom gains $0.5 e$ from the adjacent $\mathrm{Sb}$ atom.

The phonon dispersion of fluorinated- and chlorinated-ZnSb monolayers are shown in the right part of Figs. 4(a,b). In such a configuration, the fluorinated- $\mathrm{ZnSb}$ is found to exhibit dynamical stability, while the chlorinated-ZnSb is dynamically instable. One-side fluorination is sufficient for the stabilization of unoccupied orbitals of the $\mathrm{Sb}$ atoms which results in dynamical stability. In contrast, in the second scenario, in which the $\mathrm{Sb}$ atoms are saturated from both surfaces of $\mathrm{ZnSb}$ by one $\mathrm{F}$ or $\mathrm{Cl}$ atoms, the fluorinated structure is found to possess dynamical instability, while the chlorinated is dynamically stable. Notably, although the single $\mathrm{Cl}$ atom tends to be adsorbed on $\mathrm{Sb}$ atom, in fully functionalized structure it shifts to a bridge site position while $\mathrm{F}$ atoms still stay on the top of $\mathrm{Sb}$ atoms. Our calculated results demonstrate that dynamically unstable free-standing monolayer of ZnSb can be stabilized via chemical functionalization by either $\mathrm{F}$ or $\mathrm{Cl}$ atoms. In addition, $\mathrm{F}$ adsorptions on $\mathrm{Sb}$ sites possess perpendicular bonds compared to the case of $\mathrm{Cl}$ atoms while $\mathrm{Cl}$ atoms tend to bind on bridge sites. Therefore, the stabilization mechanisms are quite different for the two types of adatoms. Notice that, the $\mathrm{Cl}-\mathrm{Sb}$ bond stretching is found to be at smaller frequencies than that of the F-Sb vibrations. Such frequencies display also quite stronger F-Sb bonds in the structure as compared to the Cl-Sb bonds. The crystal
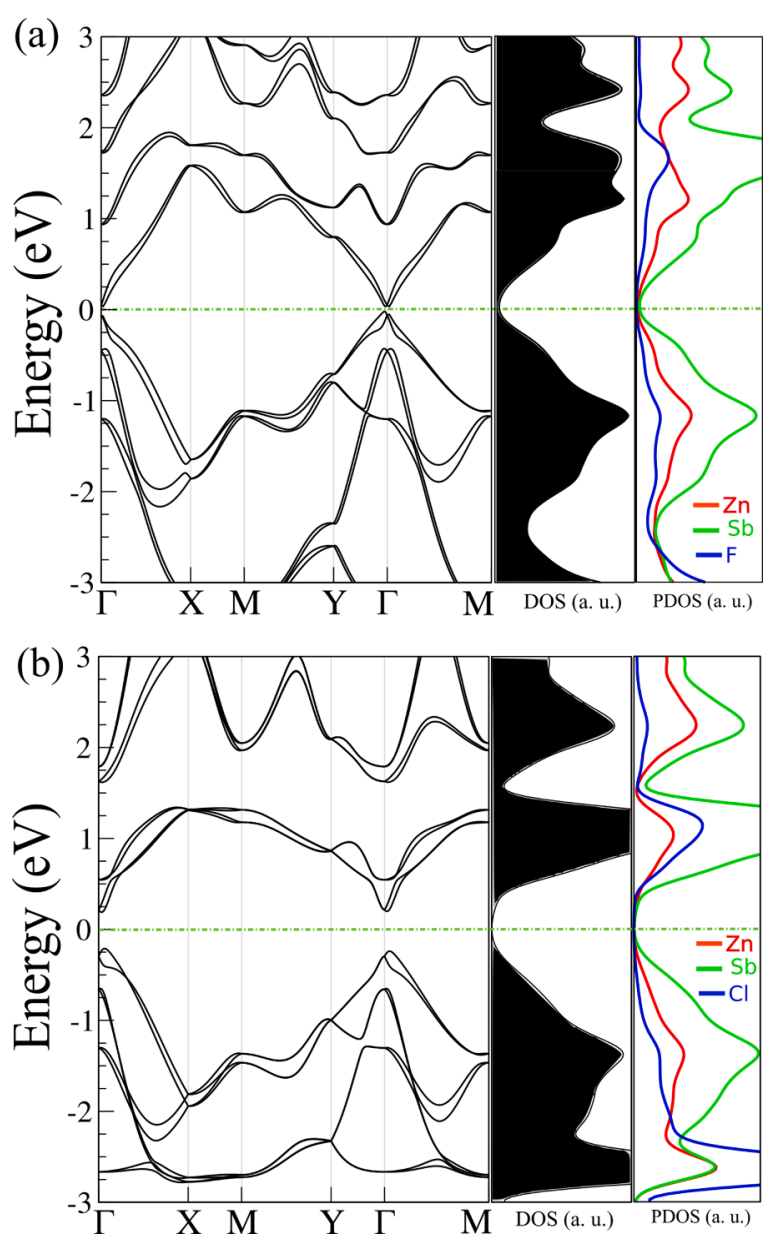

Fig. 5. The electronic band structures and its related DOS and PDOS calculated at PBE level including SOC effect for (a) fluorinated-ZnSb and (b) chlorinated$\mathrm{ZnSb}$. The zero of energy is set to the Fermi level energy. Notably, the electronic properties are presented for the dynamically stable functionalized structures.

structures and the variation of total energy of ZnSb-Cl (left) and ZnSb-F (right) around room temperature is shown in Fig. S1 of the supplementary information. our molecular dynamics simulations of the functionalized single layers revealed that while the chemically adsorbed atoms are stable on the surface at room temperature, $\mathrm{Zn}$ and $\mathrm{Sb}$ atoms have a tendency to distortion. In the case of $\mathrm{ZnSb}-\mathrm{Cl}$ the single layer is much more distorted, while the $\mathrm{ZnSb}-\mathrm{F}$ is more stable.

The electronic band structure, DOS and PDOS with corresponding SOC for fluorinated-ZnSb and chlorinated-ZnSb, respectively, are presented in Figs. 5(a,b). On the other hand, the electronic properties of fluorinated and chlorinated $\mathrm{ZnSb}$ structures reveal their semiconducting behavior in contrast to metallic ZnSb. The direct band gaps of fluorinated and chlorinated $\mathrm{ZnSb}$ are calculated to be 0.06 and $0.5 \mathrm{eV}$, respectively. In both of the structures, it is shown that the valence and the conduction band edges are mostly dominated by the Sb-states while the $\mathrm{Cl}$ atoms also have contributions to the conduction band states. Due to underestimation of the electronic band gap values of semiconductors in the PBE functional, the screened hybrid functional HSE06 were also used to provide more accurate estimations for the band-gap values of these materials as depicted in Fig. 5 by the blue line. It is conspicuous the HSE06 functional does not change the sort of direct semiconducting band gap in fluorinated-ZnSb and chlorinated-ZnSb monolayers. According to the HSE06 results, the direct band-gaps of the fluorinated$\mathrm{ZnSb}$ and chlorinated-ZnSb monolayers at $\Gamma$-point were predicted to be 1 and $1.4 \mathrm{eV}$, respectively. Our results also showed the hole effective mass of the fluorinated-ZnSb along $\Gamma \longrightarrow \mathrm{Y}$ is $0.41 m_{e}{ }^{*}$ while it is 0.55 

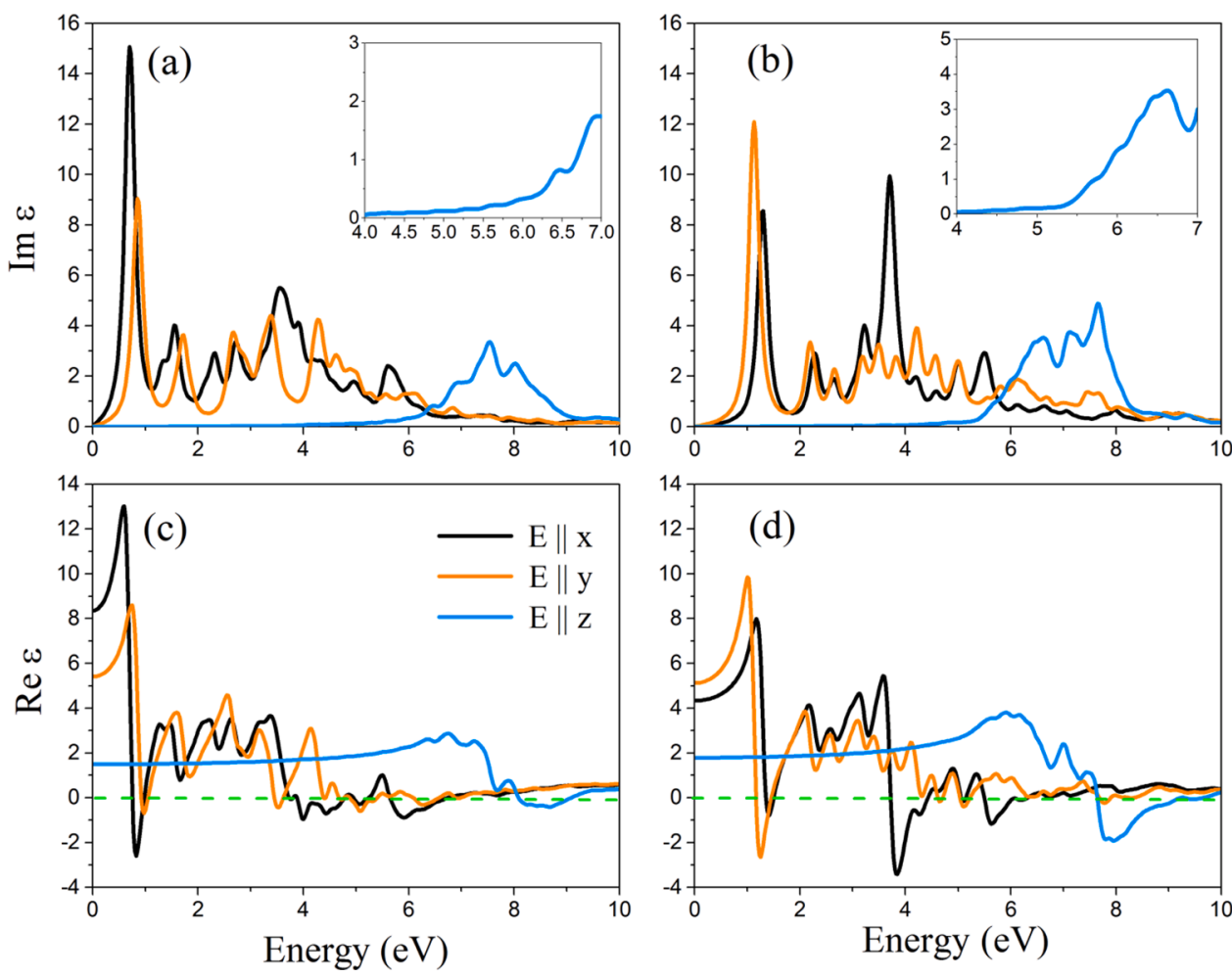

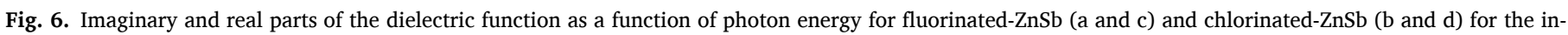
plane and out-of-plane polarizations. Insets show the $\operatorname{Im}(\varepsilon)$ at the frequency range of 4-7 eV.

$m_{e}{ }^{*}$ for chlorinated-ZnSb along $\Gamma \longrightarrow \mathrm{X}$. The effective electron masses of the fluorinated-ZnSb were obtained to be $0.36,0.22$ and $0.46 m_{e}{ }^{*}$ along $\Gamma \longrightarrow \mathrm{X}, \Gamma \longrightarrow \mathrm{Y}$ and $\Gamma \longrightarrow \mathrm{M}$, respectively. The corresponding values of the chlorinated-ZnSb along the same directions are $0.20,0.07$ and 0.27 $m_{e}{ }^{*}$. These light electron and hole effective masses lead to high carriers mobility in the nanostructures investigated.

\section{Optical properties}

We have also investigated the optical response of the novel functionalized-ZnSb systems using the RPA method constructed over the HSE06. The in-plane (E $\| \mathrm{x}$ and $\mathrm{E} \| \mathrm{y})$ and out-of-plane polarized directions with imaginary and real parts of the dielectric function have been calculated and the acquired results are illustrated in Fig. 6. The insets show the $\operatorname{Im}(\varepsilon)$ at the frequency range of 4-7 eV. Obviously, the optical spectra of both strained systems are highly anisotropic for the light polarizations along the $\mathrm{x}$-, $\mathrm{y}$ - and the $\mathrm{z}$-axis. For the fluorinated-ZnSb, the first absorption peak of $\operatorname{Im}(\varepsilon)$ is at $0.69,0.84$ and $5.80 \mathrm{eV}$ for $\mathrm{E} \| \mathrm{x}$, $\mathrm{E} \| \mathrm{y}$ and $\mathrm{E} \| \mathrm{z}$, respectively. The corresponding values of the chlorinated$\mathrm{ZnSb}$ along the same directions are $1.24,1.13$ and $5.69 \mathrm{eV}$. These results indicate that the first absorption peaks of $\operatorname{Im}(\varepsilon)$ of both monolayers are in the infrared range along the in-plane polarizations and are related to $\pi$ $\longrightarrow \pi^{*}$ transitions. The mean two peaks which are related $\pi \longrightarrow \sigma^{*}$ and $\sigma$ $\longrightarrow \pi^{*}$ occur in between 7 and $8 \mathrm{eV}$ energy range along the out-of-plane direction. While real part of the dielectric constant has been found zero, the static dielectric constants of the fluorinated-ZnSb monolayer were calculated as follows 8.33, 5.49 and 1.41 for $\mathrm{E}\|\mathrm{x}, \mathrm{E}\| \mathrm{y}$ and $\mathrm{E} \| \mathrm{z}$, respectively. The corresponding values of the effective electron masses for chlorinated-ZnSb along the same directions are 0.20, 0.07 and $0.27 \mathrm{~m}_{e}{ }^{*}$.

In the Drude model the plasma frequencies are defined by the roots of $\operatorname{Re}(\varepsilon)$ with $\mathrm{x}=0$ line [79-81], and were calculated for both systems. The corresponding values of the first plasma frequencies of fluorinated-ZnSb along the $\mathrm{x}$-y-axes are at 0.76 and $0.95 \mathrm{eV}$, respectively, while they are at 1.39 and $1.17 \mathrm{eV}$ for chlorinated-ZnSb monolayer which are related to

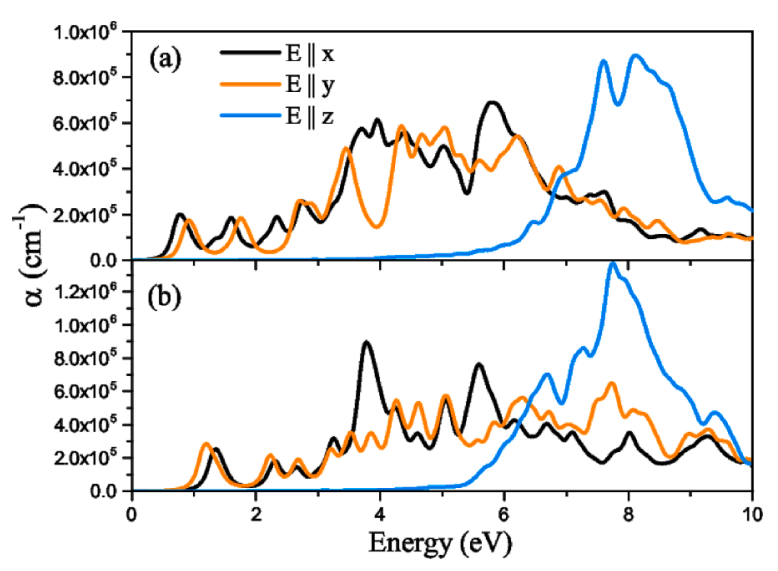

Fig. 7. Optical absorption spectra as a function of photon energy of fluorinated$\mathrm{ZnSb}$ (a) and chlorinated-ZnSb (b) for the in-plane and out-of-plane polarizations.

the $\pi$ electron plasmon peak. The plasma frequency along the z-axes occurs at an energy 8.11 and $7.65 \mathrm{eV}$ for the fluorinated-ZnSb and the chlorinated-ZnSb monolayers, respectively, which are related to the $\pi+$ $\sigma$ electron plasmon peaks.

The absorption coefficient $\alpha$ of both studied systems along all polarizations are plotted in Fig. 7. The first absorption peak of the fluorinated-ZnSb along $\mathrm{E}\|\mathrm{x}, \mathrm{E}\| \mathrm{y}$ and $\mathrm{E} \| \mathrm{z}$ occurs at energy of $0.77,0.91$ and $5.06 \mathrm{eV}$, respectively, while the corresponding values of the chlorinated-ZnSb are 1.36, 1.19 and $5.40 \mathrm{eV}$. The first absorption peaks of these novel monolayers along the in-plane polarizations are located in the infrared range (IR), while they are in the middle ultraviolet range $(U V)$ for out-of-plane polarization. The second absorption peaks of both monolayers occur at energy range between 1.6 and $2.3 \mathrm{eV}$ along the inplane polarization, which is a desirable property for practical 
applications in optoelectronic devices in the visible spectral range. The main absorption peak of the aforementioned systems for in-plane polarizations situate at the energy range between 4.0 and $6.0 \mathrm{eV}$ while it occurs around at $8.0 \mathrm{eV}$ for out-of-plane polarization. In general, the inplane optical anisotropy of these novel nanosheets is highly desirable for design of novel optoelectronic nanodevices that exploit anisotropic properties, such as e.g., polarization-sensitive photodetectors [82,83].

\section{Conclusion}

In summary, we have explored the structural, electronic and optical properties of the $\mathrm{ZnSb}$ monolayer and its functionalized derivatives by using DFT calculations. Phonon band dispersions have revealed the monolayer of ZnSb exhibits dynamical instability in its free-standing form and has a metallic behavior. We have demonstrated that $\mathrm{ZnSb}$ monolayer can be stabilized via surface chemical functionalization with $\mathrm{F}$ and $\mathrm{Cl}$ atoms. Semi-fluorinated and fully-chlorinated $\mathrm{ZnSb}$ monolayers are direct semiconductors with 1 and $1.4 \mathrm{eV}$ band gap values, respectively as well as they have very light electron and hole effective masses. Moreover, from the optical response data of the functionalized $\mathrm{ZnSb}$, we can see the first absorption peaks along the in-plane polarization reveal that the functionalized ZnSb monolayers can absorb $I R$ and visible light, suggesting their prospect toward applications in optoelectronics and nanoelectronics. Our results revealed that dynamically unstable freestanding ZnSb monolayer can be stabilized via fluorination and chlorination such that the metallic $\mathrm{ZnSb}$ monolayer turns into semiconducting monolayer crystal.

\section{Declaration of competing interest}

The authors declare that they have no known competing financial interests or personal relationships that could have appeared to influence the work reported in this paper.

\section{Acknowledgments}

This work was supported by the National Research Foundation of Korea (NRF) grant funded by the Korea government (MSIT)(NRF2017R1A2B2011989). Computational resources were provided by the Flemish Supercomputer Center (VSC). M.Y. is supported by the Flemish Science Foundation (FWO-Vl) by a postdoctoral fellowship. (EXC 2122, Project ID 390833453).

\section{Supplementary material}

Supplementary material associated with this article can be found, in the online version, at $10.1016 / \mathrm{j}$.susc. 2020.121796

\section{References}

[1] S.K. Novoselov, A.K. Geim, S.V. Morozov, D. Jiang, Y. Zhang, S.V. Dubonos, I. Grigorieva, A.A. Firsov, Electric field effect in atomically thin carbon films, Science 306 (666) (2004).

[2] A.H.C. Neto, K. Novoselov, New directions in science and technology: twodimensional crystals, Rep. Prog. Phys. 74 (082501) (2011).

[3] K.F. Mak, C. Lee, J. Hone, J. Shan, T.F. Heinz, Atomically thin $\operatorname{mos}_{2}$ : a new directgap semiconductor, Phys. Rev. B 105 (136805) (2010).

[4] A. Splendiani, L. Sun, Y. Zhang, T. Li, J. Kim, C.-Y. Chim, G. Galli, F. Wang, Emerging photoluminescence in monolayer $\operatorname{mos}_{2}$, Nano Lett. 10 (1271) (2010).

[5] B. Radisavljevic, A. Radenovic, J. Brivio, V. Giacometti, A. Kis, Single-layer mos transistors, Nat. Nanotechnol. 6 (147) (2011).

[6] R.A. Gordon, D. Yang, E.D. Crozier, D.T. Jiang, R.F. Frindt, Structures of exfoliated single layers of $\mathrm{WS}_{2}, \mathrm{mos}_{2}$, and $\mathrm{mose}_{2}$ in aqueous suspension, Phys. Rev. B 65 (125407) (2002).

[7] J.N. Coleman, M. Lotya, A. O'Neill, S.D. Bergin, P.J. King, U. Khan, K. Young, A. Gaucher, S. De, R.J. Smith, I.V. Shvets, S.K. Arora, G. Stanton, H.Y. Kim, K. Lee G.T. Kim, G.S. Duesberg, T. Hallam, J.J. Boland, J.J. Wang, J.F. Donegan, J. C. Grunlan, G. Moriarty, A. Shmeliov, R.J. Nicholls, J.M. Perkins, E.M. Grieveson, K. Theuwissen, D.W. McComb, P.D. Nellist, V. Nicolosi, Two-dimensional nanosheets produced by liquid exfoliation of layered materials, Science 331 (568) (2011).
[8] J.S. Ross, P. Klement, A.M. Jones, N.J. Ghimire, J. Yan, D.G. Mandrus, T. Taniguchi, K. Watanabe, K. Kitamura, W. Yao, D.H. Cobden, X. Xu, Electrically tunable excitonic light-emitting diodes based on monolayer $\mathrm{WSe}_{2}$ pn junctions, Nat. Nanotechnol. 9 (268) (2014).

[9] H. Sahin, S. Tongay, S. Horzum, W. Fan, J. Zhou, J. Li, J. Wu, F.M. Peeters, Anomalous raman spectra and thickness-dependent electronic properties of $\mathrm{WSe}_{2}$, Phys. Rev. B 87 (165409) (2013).

[10] S. Tongay, H. Sahin, C. Ko, A. Luce, W. Fan, K. Liu, J. Zhou, Y.S. Huang, C.H. Ho, J. Yan, D.F. Ogletree, S. Aloni, J. Ji, S. Li, L. Li, F.M. Peeters, J. Wu, Monolayer behaviour in bulk res 2 due to electronic and vibrational decoupling, Nat. Comm. 5 (3252) (2014).

[11] S. Horzum, D. Cakir, J. Suh, S. Tongay, Y.S. Huang, C.H. Ho, J. Wu, H. Sahin, F. M. Peeters, Formation and stability of point defects in monolayer rhenium disulfide, Phys. Rev. B 89 (155433) (2014).

[12] B. Chen, H. Sahin, A. Suslu, L. Ding, K.M.I. Bertoni, F.M. Peeters, S. Tongay, Environmental changes in mote $_{2}$ excitonic dynamics by defects-activated molecular interaction, ACS Nano 9 (5326) (2015).

[13] S. Cahangirov, M. Topsakal, E. Akturk, H. Sahin, S. Ciraci, Two- and onedimensional honeycomb structures of silicon and germanium, Phys. Rev. Lett. 102 (236804) (2009).

[14] A. Kara, H. Enriquez, A.P. Seitsonen, L.C.L.Y. Voon, S. Vizzini, B. Aufray, H. Oughaddou, A review on silicene new candidate for electronics, Surf. Science Report. 67 (1) (2012).

[15] H. Sahin, S. Cahangirov, M. Topsakal, E. Bekaroglu, E. Akturk, R.T. Senger, S. Ciraci, Monolayer honeycomb structures of group-IV elements and III-V binary compounds: first-principles calculations, Phys. Rev. B 80 (155453) (2009).

[16] Q. Wang, Q. Sun, P. Jena, Y. Kawazoe, Potential of aln nanostructures as hydrogen storage materials, ACS Nano 3 (621) (2009).

[17] K.K. Kim, A. Hsu, X. Jia, S.M. Kim, Y. Shi, M. Hofmann, D. Nezich, J.F. RodriguezNieva, M. Dresselhaus, T. Palacios, J. Kong, Synthesis of monolayer hexagonal boron nitride on cu foil using chemical vapor deposition, Nano Lett. 12 (161) (2012).

[18] P. Tsipas, S. Kassavetis, D. Tsoutsou, E. Xenogiannopoulou, E. Golias, S.A. Giamini, C. Grazianetti, D. Chiappe, A. Molle, M. Fanciulli, A. Dimoulas, Evidence for graphite-like hexagonal aln nanosheets epitaxially grown on single crystal ag(111), Appl. Phys. Lett. 103 (251605) (2013).

[19] C. Bacaksiz, H. Sahin, H.D. Ozaydin, S. Horzum, R.T. Senger, F.M. Peeters, Hexagonal AIN: dimensional-crossover-driven band-gap transition, Phys. Rev. B 91 (085430) (2015).

[20] D.A. Chenet, O.B. Aslan, P.Y. Huang, C. Fan, A.M. van der Zande, T.F. Heinz, J. C. Hone, In-plane anisotropy in mono- and few-layer $\mathrm{r}_{2}$ probed by raman spectroscopy and scanning transmission electron microscopy, Nano Lett. 15 (5667) (2015).

[21] L. Hart, S. Dale, S. Hoye, J. Webb, D. Wolverson, Rhenium dichalcogenides: layered semiconductors with two vertical orientations, Nano Lett. 16 (1381) (2016).

[22] S. Yang, S. Tongay, Y. Li, Q. Yue, J.-B. Xia, S.-S. Li, L. Li, S.H. Wei, Layer-dependent electrical and optoelectronic responses of rese 2 nanosheet transistors, Nanoscale 6 (7226) (2014).

[23] S. Yang, S. Tongay, Q. Yue, Y. Li, B. Li, F. Lu, High-performance few-layer modoped rese 2 nanosheet photodetectors, Sci. Rep. 4 (5442) (2014).

[24] J. Chu, Y. Zhang, Y. Wen, R. Qiao, C. Wu, P. He, L. Yin, R. Cheng, F. Wang, Z. Wang, J. Xiong, Y. Li, J. He, Sub-millimeter-scale growth of one-unit-cell-thick ferrimagnetic $\mathrm{cr}_{2} \mathrm{~s}_{3}$ nanosheets, Nano Lett. 19 (2154) (2019).

[25] A.P. Balan, S. Radhakrishnan, C.F. Woellner, S.K. Sinha, L. Deng, de los, C. Reyes, B.M. Rao, M. Paulose, R. Neupane, A. Apte, V. Kochat, R. Vajtai, A.R. Harutyunyan, C.W. Chu, C.-W.G. Costin, D.S. Galvao, A.A. Marti, P.A. vanAken, O.K. Varghese, C. S. Tiwary, A.M.M.R. Iyer, P.M. Ajayan, Exfoliation of a non-van der waals material from iron ore hematite, Nat. Nanotech. 13 (602) (2018).

[26] C. Bacaksiz, M. Yagmurcukardes, F.M. Peeters, M.V. Milosevic, Hematite at its thinnest limit, 2D Mater. 7 (2) (2020).

[27] V. Kochat, A. Samanta, Y. Zhang, S. Bhowmick, P. Manimunda, S. Asif, A. S. Stender, R. Vajtai, A.K. Singh, C. Tiwary, P.M. Ajayan, Atomically thin gallium layers from solid-melt exfoliation, Sci. Adv. 4 (1701373) (2018).

[28] J. Song, H.Y. Song, Z. Wang, S. Lee, J.Y. Hwang, S.Y. Lee, L. Jouhahn, K. Dongwook, L. KyuHyong, K. Youngkuk, H.O. Sang, W.K. Sung, Creation of twodimensional layered zintl phase by dimensional manipulation of crystal structure, Sci. Adv. 5 (6) (2019).

[29] D.B. Xiong, N.L. Okamoto, H. Inui, Enhanced thermoelectric figure of merit in ptype ag-doped znsb nanostructured with $\mathrm{ag}_{3}$ sb, Scr. Mater. 69 (397) (2013).

[30] M.I. Fedorov, L.V. Prokofeva, D.A. Pshenay-Severin, A.A. Shabaldin, P. P. Konstantinov, New interest in intermetallic compound znsb, J. Elect. Mater. 43 (2314) (2014).

[31] K. Valset, P.H.M. Bottger, J. Tafto, T.G. Finstad, Thermoelectric properties of Cu doped ZnSb containing Zn3P2 particles, J. Appl. Phys. 111 (023703) (2012).

[32] J. Sottmann, K. Valset, O.B. Karlsen, J. Tafto, Synthesis and measurement of the thermoelectric properties of multiphase composites: ZnSb Matrix with Zn4Sb3, Zn3P2, and Cu5zN8, J. Elect. Mater. 42 (1820) (2013).

[33] Q. Wang, X. Qin, D. Li, T. Zou, Enhancement of thermopower and thermoelectric performance through resonant distortion of electronic density of states of N4Sb3 doped with Sm, Appl. Phys. Lett. 102 (154101) (2013).

[34] G.H. Zhu, W.S. Liu, Y.C. Lan, G. Joshi, H. Wang, G. Chen, Z.F. Ren, The effect of secondary phase on thermoelectric properties of Zn4Sb3 compound, Nano Energy 2 (1172) (2013).

[35] J.-B. Li, M.-C. Record, J.C. Tedenac, A thermodynamic assessment of the SbZn system, J. Alloys Compd. 438 (171) (2007). 
[36] F. Adjadj, E.D. Belbacha, M. Bouharkat, Differential calorimetric analysis of the binary system SbZn, J. Alloys Compd. 430 (85) (2007).

[37] K. Valset, X. Song, T.G. Finstad, A study of transport properties in Cu and P doped ZnSb, J. Appl. Phys. 117 (045709) (2015).

[38] Q. Guo, S. Luo, Improved thermoelectric efficiency in p-type ZnSb through Zn deficiency, Funct. Mater. Lett. 8 (1550028) (2015).

[39] K. Niedziolka, P. Jund, Influence of the exchange correlation functional on the electronic properties of $\mathrm{ZnSb}$ as a promising thermoelectric material, J. Elect. Mater. 44 (1540) (2015).

[40] J.O. Sofo, A.S. Chaudhari, G.D. Barber, Graphane: a two-dimensional hydrocarbon, Phys. Rev. B 75 (153401) (2007).

[41] W. Wei, T. Jacob, Strong charge-transfer excitonic effects in $\mathrm{C}_{4} \mathrm{H}$-type hydrogenated graphene, Phys. Rev. B 86 (165444) (2012).

[42] P. Cudazzo, C. Attaccalite, I.V. Tokatly, A. Rubio, Strong charge-transfer excitonic effects and the Bose-Einstein exciton condensate in graphane, Phys. Rev. Lett. 104 (226804) (2010).

[43] R. Zapata-Pena, B.S. Mendoza, A.I. Shkrebtii, Pure spin current injection in hydrogenated graphene structures, Phys. Rev. B 96 (195415) (2017).

[44] J.-C. Charlier, X. Gonze, J.P. Michenaud, First-principles study of graphite monofluoride $(\mathrm{CF})_{n}$, Phys. Rev. B 47 (16162) (1993).

[45] Y. Takagi, K. Kusakabe, Transition from direct band gap to indirect band gap in fluorinated carbon, Phys. Rev. B 65 (121103) (2002).

[46] H. Sahin, M. Topsakal, S. Ciraci, Structures of fluorinated graphene and their signatures, Phys. Rev. B 83 (115432) (2011).

[47] H. Yang, M. Chen, H. Zhou, C. Qiu, J. Hu, F. Yu, W. Chu, S. Sun, L. Sun, Preferentia and reversible fluorination of monolayer graphene, J. Phys. Chem. C 115 (16844) (2011).

[48] R.R. Nair, W. Ren, R. Jalil, I. Riaz, V.G. Kravets, L. Britnell, P. Blake, F. Schedin, A. S. Mayorov, S. Yuan, M.I. Katsnelson, H.-M. Cheng, W. Strupinski, L.G. Bulusheva, A.V. Okotrub, I.V. Grigorieva, A.N. Grigorenko, K.S. Novoselov, A.K. Geim, Fluorographene: a two dimensional counterpart of teflon, Small 6 (2877) (2010).

[49] M. Yagmurcukardes, Monolayer fluoro-InSe: formation of a thin monolayer via fluorination of InSe, Phys. Rev. B 100 (024108) (2019).

[50] V. Sreepal, M. Yagmurcukardes, S.K. Vasu, D.J. Kelly, S.F.R. Taylor, V.G. Kravets, Z. Kudrynskyi, Z.D. Kovalyuk, A. Patan, A.N. Grigorenko, S.J. Haigh, C. Hardacre, L. Eaves, H. Sahin, A.K. Geim, F.M. Peeters, R.R. Nair, Two-dimensional covalen crystals by chemical conversion of Thin van der waals materials, Nano Lett. 19 (6475) (2019).

[51] J.-C. Charlier, X. Gonze, J.P. Michenaud, First-principles study of graphite monofluoride $(\mathrm{CF})_{n}$, Phys. Rev. B 47 (16162) (1993).

[52] A. Bafekry, M. Yagmurcukardes, M. Shahrokhi, M. Ghergherehchi, D. Kim, M. Ghergherehchi, Monolayer and bilayer zinc antimonide (ZnSb) honeycomb structures : a first-principles study, Appl. Surf. Sci. 504 (2020) 148289.

[53] A. Bafekry, C. Stampfl, C. Nguyen, M. Ghergherehchi, B. Mortazavi, Tunable electronic properties of the dynamically stable layered mineral $\mathrm{pt}_{2} \mathrm{HgSe}_{3}$ (Jacutingaite), Phys. Chem. Chem. Phys. 22 (2020) 24471.

[54] Y. Takagi, K. Kusakabe, Transition from direct band gap to indirect band gap in fluorinated carbon, Phys. Rev. B 65 (121103) (2002).

[55] A. Bafekry, C. Stampfl, Band-gap control of graphenelike borocarbonitride g-BC6N bilayers by electrical gating,, Phys. Rev. B 102 (19) (2020) 195411.

[56] A. Bafekry, M.M. Fadlallah, C. Nguyen, D. Gogova, Vertical two-dimensional layered conjugated porous organic network structures of polybenzimidazobenzophenanthroline (BBL): afirst-principles study, Appl. Phys. Lett. 117 (2020) 233101.

[57] R.R. Nair, W. Ren, R. Jalil, I. Riaz, V.G. Kravets, L. Britnell, P. Blake, F. Schedin, A. S. Mayorov, S. Yuan, M.I. Katsnelson, H.-M. Cheng, W. Strupinski, L.G. Bulusheva, A.V. Okotrub, I.V. Grigorieva, A.N. Grigorenko, K.S. Novoselov, A.K. Geim, Fluorographene: a two dimensional counterpart of teflon, Small 6 (2877) (2010).

[58] A. Bafekry, F. Shojaei, D.M. Hoat, M. Shahrokhi, M. Ghergherehchi, C. Nguyen, Two-dimensional silicon bismotide (sibi) monolayer with a honeycomb-like lattice: first-principles study of tuning the electronic properties, RSC Adv 10 (53) (2020) 31894-31900.
[59] A. Bafekry, C. Nguyen, M. Obeid, M. Ghergherehchi, Modulating the electro-optical properties of doped C3N monolayer and graphene bilayer via mechanical strain and pressure, New J. Chem 44 (36) (2020) 15785-15792.

[60] H. Sahin, M. Topsakal, S. Ciraci, Structures of fluorinated graphene and their signatures, Phys. Rev. B 83 (115432) (2011).

[61] A. Bafekry, F. Shojaei, M.M. Obeid, M. Ghergherehchi, C. Nguyen, M. Oskouian, The mechanical, electronic, optical and thermoelectric properties of twodimensional honeycomb-like of XSb ( $\mathrm{X}=\mathrm{Si}, \mathrm{Ge}, \mathrm{Sn})$ monolayers: a first-principles calculations, RSC Adv 10 (52) (2020) 31526.

[62] H. Yang, M. Chen, H. Zhou, C. Qiu, L. Hu, F. Yu, W. Chu, S. Sun, L. Sun, Preferential and reversible fluorination of monolayer graphene, J. Phys. Chem. C 115 (16844) (2011).

[63] H. Sahin, S. Ciraci, Chlorine adsorption on graphene: chlorographene, J. Phys. Chem. C 116 (24075) (2012).

[64] M. Yang, L. Zhou, J. Wang, Z. Liu, Z. Liu, Evolutionary chlorination of graphene: from charge-transfer complex to covalent bonding and nonbonding, J. Phys. Chem. C 116 (844) (2011).

[65] H.L. Poh, P. Simek, Z. Sofer, M. Pumera, Halogenation of graphene with chlorine, bromine, or iodine by exfoliation in a halogen atmosphere, Chem.Eur. J. 19 (2655) (2013).

[66] B. Li, L. Zhou, D. Wu, H. Peng, K. Yan, Z. Liu, Y. Zhou, Photochemical chlorination of graphene. ACS Nano 5 (5957) (2011).

[67] Y.-Z. Tan, B. Yang, K. Parvez, A. Narita, S. Osella, D. Beljonne, X. Feng, K. Mullen, Atomically precise edge chlorination of nanographenes and its application in graphene nanoribbons, Nat. Commun. 4 (2646) (2013).

[68] J.P. Perdew, K. Burke, M. Ernzerhof, Generalized gradient approximation made simple, Phys. Rev. Lett. 77 (3865) (1996).

[69] G. Kresse, J. Furthmuller, Efficient iterative schemes for ab initio total-energy calculations using a plane-wave basis set, Phys. Rev. B 54 (11169) (1996).

[70] G. Kresse, J. Hafner, Ab initio molecular dynamics for liquid metals, Phys. Rev. B 47 (558) (1993).

[71] G. Kresse, J. Hafner, Ab initio molecular-dynamics simulation of the liquid-metal amorphous-semiconductor transition in germanium, Phys. Rev. B 49 (14251) (1994).

[72] J. Heyd, G.E. Scuseria, M. Ernzerhof, Hybrid functionals based on a screened coulomb potential, J. Chem. Phys. 118 (8207) (2003).

[73] S.J. Grimme, Semiempirical GGA type density functional constructed with a long range dispersion correction, Comput. Chem. 27 (1787) (2006).

[74] G. Henkelman, A. Arnaldsson, H. Jonsson, A fast and robust algorithm for Bader decomposition of charge density, Comput. Mater. Sci. 36 (354) (2006).

[75] H.J. Monkhorst, J.D. Pack, Special points for Brillouin-zone integrations, Phys. Rev. B 13 (12) (1976).

[76] D. Alfe, PHON: A program to calculate phonons using the small displacement method, Comput. Phys. Commun. 180 (2622) (2009).

[77] M. Gajdos, K. Hummer, G. Kresse, J. Furthmller, F. Bechstedt, Linear optica properties in the projector-augmented wave methodology, Phys. Rev. B 73 (045112) (2006).

[78] J. Heyd, G.E. Scuseria, Efficient hybrid density functional calculations in solids: assessment of the Heyd Scuseria Ernzerhof screened Coulomb hybrid functional, J. Chem. Phys. 121 (1187) (2004).

[79] F. Wooten, Optical properties of solids, Academic press, 2013.

[80] M. Shahrokhi, S. Naderi, A. Fathalian, Ab initio calculations of optical properties of B2C graphene sheet, Solid State Commun. 152 (1012) (2012).

[81] M. Shahrokhi, C. Leonard, Tuning the band gap and optical spectra of silicon-doped graphene: many-body effects and excitonic states, J. Alloys Compd. 693 (1185) (2017).

[82] H. Yuan, X. Liu, F. Afshinmanesh, W. Li, G. Xu, J. Sun, B. Lian, A.G. Curto, G. Ye, Y. Hikita, Z. Shen, Polarization-sensitive broadband photodetector using a black phosphorus vertical pn junction, Nat. Nanotechnol. 10 (707) (2015).

[83] M. Shahrokhi, Can fluorine and chlorine functionalization stabilize the graphene like borophene? Comput. Mater. Sci. 156 (56) (2019). 